\title{
Evoking and Reducing Mental Contamination in Female Perpetrators of an Imagined Non-Consensual Kiss
}

Katie Waller and Mark J. Boschen*

School of Applied Psychology, Griffith University, Southport Australia

* Corresponding Author: Dr Mark Boschen, School of Applied Psychology, Griffith University, Parklands Drive, Southport, Australia. Ph: +61 7 55528283, m.boschen@griffith.edu.au 


\begin{abstract}
Background and Objectives: Mental contamination refers to feelings of internal dirtiness that can arise without physical contact with a contaminant. Previous research has demonstrated that contamination-related feelings and subsequent washing behaviours can be evoked by engaging in an imaginal task involving a non-consensual kiss. We sought to test the efficacy of neutralisation behaviours such as washing on experimentally induced mental contamination.
\end{abstract}

Methods: The current study used a female undergraduate sample $(N=80)$ to act as the perpetrator of an imagined non-consensual kiss of a 14-year old boy, to examine whether mental contamination would be evoked, and whether neutralisation would be effective.

Results: Mental contamination was successfully evoked in using the imaginal task. None of the neutralisation strategies (physical washing, mental washing, atonement) was more effective than a control group in reducing mental contamination. Groups using physical washing completed the experiment with higher levels of negative emotions than the control group, suggesting specific deleterious impact of this neutralisation behaviour.

Limitations: The use of a non-clinical sample, as well as a uniform mental contamination method (rather than one specifically tailored to each participant) are limitations of the current study.

Conclusions: Mental contamination is not reduced by a range of neutralisation strategies, and physical washing may have further negative effects such as increased negative emotion.

KEYWORDS: Mental contamination; OCD; compulsive cleaning 


\subsection{Introduction}

Mental contamination may contribute to poor treatment outcomes for individuals with obsessive-compulsive disorder (OCD), where cleaning compulsions are a significant facet of their presentation (Fisher \& Wells, 2005; Foa et al., 2005; Rachman, 2004, 2006). With a lifetime prevalence of approximately 1.3\% (Somers, Goldner, Waraich, \& Hsu, 2006), OCD can lead to significant functional impairment (Markarian et al., 2010), physical complications (Drummond et al., 2012), and reduced quality of life (Fontenelle et al., 2010). Despite ongoing research (Boschen, 2008), technological developments (Lind, Boschen, \& Morrissey, 2013), and effective treatments for even the most severe presentations (Boschen \& Drummond, 2012; Boschen, Drummond, \& Pillay, 2008), many individuals do not respond to treatment (Fisher \& Wells, 2005), and predicting those who will respond has proven difficult (Boschen, Drummond, Pillay, \& Morton, 2010; Farrell \& Boschen, 2011). Approximately 30-50\% of individuals with OCD have a fear of contact contamination (Foa et al., 1995; Rachman \& Hodgson, 1980), and cleaning is the second most common compulsion. Patients presenting with washing compulsions stemming from contamination fear may show poorer response to therapies such as exposure and response prevention (ERP; Coehlo \& Whittal, 2001) or pharmacotherapy (Alarcon, Libb, \& Spitler, 1993).

Mental contamination (Rachman, 2004; 2006; Shafran, Thordarson, \& Rachman, 1996) may contribute to the intractable nature of both contamination-related feelings and of washing compulsions. Mental contamination is described as "a sense of internal un-cleanness which can and does usually arise and persist regardless of the presence or absence of external, observable dirt” (Rachman, 1994, p.311). It can be triggered by an association with impurity, immorality, unpleasant memories, and by aversive events such as sexual assault (Fairbrother, Newth, \& Rachman, 2005; Herba \& Rachman, 2007; Rachman, 2004), perceived mistreatment (Warnock-Parkes, Salkovskis, \& Rachman, 2012), betrayal, or shameful acts (Coughtrey, 
Shafran, Lee, \& Rachman, 2012). Poor outcomes in the treatment of washing compulsions may be explained by an undifferentiated approach to treating contact contamination fear and mental contamination. Until the last decade, there has been little research into the phenomenon of mental contamination (Coughtrey, Shafran, Lee, \& Rachman, 2013; Warnock-Parkes et al., 2012). ERP using stimuli that trigger a sense of physical (but not mental) contamination may yield suboptimal treatment outcomes for some individuals where mental contamination is important.

As the hands represent a common source of contact contamination, mental contamination may lead to attempts to localise the contamination to the hands, as would be done after touching something contaminated (Fairbrother \& Rachman, 2004; Rachman, 1994). However, mental contamination has been observed to persist after washing (Coughtrey et al., 2012; Fairbrother \& Rachman, 2004; Rachman, 1994). Physical washing may be ultimately ineffective, as washing does not reduce less tangible internal feelings of dirtiness.

The first study to illustrate that contamination-related feelings could be evoked by a mental event was reported by Fairbrother and Rachman (2004). It revealed that asking female sexual assault victims to deliberately recall their assault re-elicited negative emotions (e.g., anxiety, distress and dirtiness) and an urge to wash, with one-fifth of the participants spontaneously engaging in physical washing. This finding inspired a model (Fairbrother et al., 2005) proposing the necessary groups for mental contamination: According to this model, the feelings of contamination can be triggered with or without physical contact and endure beyond the time in which the person was exposed to the trigger stimulus. These feelings of contamination lead to unpleasant emotions and a subsequent urge to wash, which is largely ineffective. Additionally, mental contamination is proposed to often include a moral component. These criteria can be used to assess mental contamination in clinical and nonclinical samples. 
The "dirty kiss" method was created to evoke mental contamination in female nonclinical samples. In this method participants listen to an audio recording describing a party scene in which the participant becomes the victim of an enforced, non-consensual kiss, or a control audiotape involves a consensual kiss. Participants are asked to listen to the recording and imagine themselves in the scene depicted, as if the events are actually occurring. A series of studies has established that this "dirty kiss” method is effective in evoking feelings of mental contamination in comparison to other control tasks (Elliott \& Radomsky, 2009a;b; Elliott \& Radomsky, 2012; Fairbrother et al., 2005; Herba \& Rachman, 2007; Rachman, Radomsky, Elliott, \& Zysk, 2012). Most of these used variables measured by the Mental Contamination Report (MCR; Herba \& Rachman, 2007), specifically, feelings of dirtiness, urge to wash, and negative emotions. In all of the aforementioned studies, a number of participants in the non-consensual conditions engaged in spontaneous washing behaviours to alleviate feelings caused by the recorded instructions. None of this previous research has examined these washing behaviours further. Zhong and Liljenquist (2006) found that handwashing after recalling an immoral deed from the past reduced negative emotions (e.g., disgust, regret, shame). However, this result was not replicated in a later study with a larger sample size (Fayard, Bassi, Bernstein, \& Roberts, 2009). In this study, individuals who had recalled an unethical deed were no more likely to choose an antibacterial hand cleanser as a free gift than those participants who recalled an ethical deed. In this study, participants also experienced no reduction in negative moral emotions (e.g., guilt) from cleaning.

Outside the laboratory, a qualitative study based on thematic analysis of semi-structured interview data, documented that 15 of 20 OCD patients with a fear of contamination reported that washing themselves was moderately effective in removing feelings of internal dirtiness and contamination (Coughtrey et al., 2012). The co-occurrence of both physical and mental contamination in the sample may explain the reported effectiveness of physical washing. 
However, if there is a link between physical washing and internal purity, the mechanisms underlying this relationship remain unclear.

In addition to research involving victims of non-consensual acts, research has also examined participants as perpetrators of non-consensual acts. In a sample of violent male offenders (Evans, Ehlers, Mezey, \& Clark, 2007), violent crimes led to a psychologically traumatic experience for the perpetrator through the acquisition of intrusive images and intense negative emotions (Evans et al., 2007). To experimentally investigate the effects of committing a non-consensual act, the "dirty kiss" method was altered to use a male sample acting as the perpetrator of the kiss (Rachman et al., 2012). In this study, the victim was the younger sister of a friend, thereby also introducing the theme of betrayal, also suggested as an important component in mental contamination (Warnock-Parkes et al., 2012). The study supported the major hypothesis, that perpetrators of the non-consensual kiss would experience feelings of mental contamination. Washing behaviours were reported, however it remained unclear whether the negative emotions evoked by the task were thereby reduced.

\subsection{The Current Study: Aims and Hypotheses}

We aimed to extend on the experiment conducted by Rachman et al. (2012) by examining the effects of physical washing and in addition, atonement on contaminationreduction in female perpetrators of the non-consensual kiss. Our first aim was to examine the non-traditional role of the female as the perpetrator of the non-consensual kiss. We sought to strengthen the "perpetrator-effect” using a task that involved a non-consensual kiss with a 14year old boy, which is recognised to go against sex-role and age related norms (Kenrick, Keefe, Gabrielidis, \& Cornelius, 1996; Stewart, Stinnett, \& Rosenfeld, 2000). Further, the male victim is positioned as the younger brother of the perpetrator's friend, introducing the previously examined theme of betrayal (Rachman et al., 2012). 
The second aim of this research was to examine the effects that different neutralising behaviours would have on mental contamination. This was done by incorporating a physical mouth-washing exercise, and also an equivalent mental (i.e., imaginal) mouth-washing exercise in order to account for the potential confounding effects of using a physical task in an experiment measuring a cognitive construct. As per the earlier discussed model of mental contamination (Fairbrother et al., 2005), both washing tasks were predicted to be equally ineffective in reducing contamination-related feelings compared to a control condition.

Experimentally reducing internal “dirty” feelings has remained relatively unexplored in current literature. Mental contamination has been reduced by receiving post-“dirty kiss” information which positions the perpetrator in a favourable light (Elliott \& Radomsky, 2010); however this method is unlikely to translate to real-world scenarios involving a true perpetrator. In an experiment where the participant is in the role of perpetrator of the nonconsensual kiss, however, this changing in the appraisal of the perpetrator (i.e., the self) may be facilitated by atonement behaviour, in which the individual makes some amends for their perceived misdeeds. Incorporating these findings, the current study also included an experimental condition in which the participant made a reappraisal of their character after atoning for their transgression.

A series of a priori hypotheses were proposed:

1. Mental contamination will be evoked in the absence of a physical stimulus using the non-consensual “dirty kiss” task, as measured by an increase in feelings of dirtiness, urge to wash, internal negative emotions and external negative emotions.

2. Neutralisation behaviours (physical washing, mental washing, atonement) will be ineffective in reducing perceived mental contamination, compared to the control condition. 
3. Where neutralisation efforts are ineffective, this will be associated with an increase in negative (internal and external) emotions, after controlling for initial levels of negative emotions at the beginning of the experiment.

\subsection{Method}

\subsection{Participants}

The sample consisted of 80 female, first year psychology students who participated in exchange for partial course credit. Random allocation resulted in 19 people in the mental washing group, 19 in the physical washing group, 20 in the atonement task group, and 22 in the control group. Ages ranged from 15 to 60 years old $(M=23.72, S D=9.93$, see Table 1$)$.

\subsection{Materials}

\subsubsection{Depression Anxiety Stress Scales - Short form (DASS-21; Lovibond \&} Lovibond, 1995). This 21-item self-report scale measures symptoms of depression, anxiety and stress experienced over the past week on a 4-point Likert scale. Internal reliability in the current study was acceptable for the depression $(\alpha=.79)$, the anxiety $(\alpha=.72)$ and stress $(\alpha=$ .78) subscales.

2.2.2 Obsessive Compulsive Inventory - Revised (OCI-R; Foa et al., 2002). This selfreport scale contains 18-items measuring the distress associated with obsessive-compulsive symptoms across six subscales of checking, washing, hoarding, obsessing, cleaning, and neutralising on a 5-point Likert scale. Internal reliability in the current study was good for the overall instrument $(\alpha=.84)$, and the washing subscale $(\alpha=.85)$.

2.2.3 Mental Contamination Report (MCR; Herba \& Rachman, 2007). The MCR is a 29-item questionnaire developed to be used in conjunction with the "dirty kiss" audiotape to assess three indices: feelings of dirtiness, urges to wash, and negative emotions on a 5-point scale from 1 (Not at all) to 5 (Very much). It also includes three manipulation check questions 
(e.g., How easy was it to imagine the scenario in your mind?). The current study used three variations of the MCR to avoid repeating irrelevant questions over three administrations. Each participant's urge to wash was assessed by averaging their urge to engage in the five washing behaviours from Question 4 of the MCR. In line with previous research (Elliott \& Radomsky, 2009a; 2009b; Elliott \& Radomsky, 2012) the negative emotions associated with mental contamination were separated into two components: internal negative emotions (mean of ashamed/guilty, humiliated, afraid, sad, and cheap/sleazy) and external negative emotions (mean of distressed/anxious, disgusted by your behaviour, and angry).

2.2.4 "Dirty Kiss" audio recording. The script was based closely on the Rachman et al. (2012) script which was successful in evoking mental contamination in a male perpetrator sample. Changes were made to make the recording appropriate for a female as the perpetrator of the kiss. The audio was 3.5 minutes in duration, and was narrated by a male voice which instructed the participant to imagine forcefully kissing their friend's 14-year old younger brother at a party before getting caught by the friend.

The four experimental task recordings were presented using a male-narrated MP3 format audio file, with each recoding approximately 90 seconds in duration. The control group was instructed to imagine standing on a busy street corner watching the cars go past. This task was intended to be emotionally neutral. The atonement group listed to an audio recording instructing them to imagine re-entering the scenario where the "dirty kiss" audio ended. In this recording, they leave the party, sit on a park bench, and reappraise the situation, realising that the kiss was "out of character" for them. Following this, they are able to view themselves in a restored moral light. Finally, they re-enter the party and apologise for their behaviour. ${ }^{1}$ The physical washing group performed a physical task. Their recording instructed them to utilise $20 \mathrm{ml}$ of mouthwash located in a plastic, transparent cup on the desk, and to gargle the mouthwash for 25 seconds, then spit it back into the cup. Similarly, the participants in the 
mental washing group heard audio that instructed them to imagine themself in a bathroom gargling strong, mint-flavoured mouthwash for 25 seconds.

\subsection{Procedure}

The experiment was conducted in a small room with two computers, allowing two participants to be tested simultaneously. Participants could not view or interact with each other during the experiment. Other distractions were minimised. Participants were initially screened to ensure that they would feel comfortable imagining kissing a young male, and were informed that some people who have experienced a sexual trauma in the past may feel less comfortable in participating. No students declined participation or withdrew before the end of the experiment. Participants were then randomly assigned to one of the four groups (mental washing, physical washing, atonement, or control). Participants completed consent procedures, demographic details, and baseline questionnaires (DASS-21, OCI-R, MCR) at their desk ("baseline”). Participants were then instructed to put on headphones attached to the computer, at which time they heard the "dirty kiss” audiotape. Participants then completed the second measurement of the MCR (“time 2”). After this, the experimental task began where procedures differed based on assigned condition (see the description of the tasks above). This was followed by the administration of the post-test MCR (“time 3”). Participants were then debriefed.

\subsection{Results}

A series of mixed-factorial ANOVA was used to examine the effects of time (within subjects; baseline, time 2, and time 3) and group (between subjects; physical washing, mental washing, atonement and control) on subjective ratings of mental contamination. Each ANOVA used one dependent variable of interest: feelings of dirtiness, urge to wash, internal negative 
emotions, or external negative emotions. Feelings of dirtiness/uncleanness scores were based on Question 2 from the MCR.

\subsection{Baseline Equivalence}

One-way ANOVAs revealed no significant group differences in age or psychopathology measures at baseline (all Fs $<1.20$, ps $>$.315), with the exception of the DASS-21 depression subscale $\left(F_{(3,76)}=3.294, p=.025\right.$, where the atonement group reported significantly higher depression symptoms than participants in the control condition $\left(M_{\text {diff }}=\right.$ 4.35, $p=.006, d=0.81$, see Table 1 ). All analyses reported here were also run using baseline depression scores as a covariate, but as this did not change the significance of any result, results without this covariate are reported throughout.

\subsection{Preliminary Analyses}

The vividness with which participants imagined the "dirty kiss" scenario was assessed at Time 2 by averaging their scores on two items: "How easy was it to imagine the scenario in your mind?" and "How clear/vivid was the imagined scenario?” There were no significant differences being found between groups, $F_{(3,75)}=0.47 ; p=.70$, and the average vividness rating being between "fairly vivid" and "quite vivid” $(M=3.17, S D=1.22)$.

Prior to specific hypothesis testing, and to protect against inflated Type I error from multiple comparisons, we analysed a 3 (Time) $\times 4$ (Group) ANOVA for each of the four dependent variables. Significant Time $\times$ Group interactions were found for dirtiness $\left(F_{(6,152)}=\right.$ 4.67, $\left.p<.001, \eta_{\mathrm{p}}{ }^{2}=.16\right)$, internal negative emotions $\left(F_{(6,152)}=3.10, p<.001, \eta_{\mathrm{p}}{ }^{2}=.17\right)$, and external negative emotions $\left(F_{(6,152)}=2.73, p=.001, \eta_{\mathrm{p}}{ }^{2}=.11\right)$, while the interaction approached significance for urge to wash $\left(F_{(6,152)}=1.28, p=.08, \eta_{\mathrm{p}}{ }^{2}=.07\right)$. The breakdown of these interactions are detailed with regard the earlier hypotheses in the sections below.

\subsection{Hypothesis One - Evoking Mental Contamination}


To test whether mental contamination was evoked, 2 (Time) $\times 4$ (Group) ANOVAs were computed to examine the effects of time (baseline, time 2) and group (physical washing, mental washing, atonement, control) on perceived mental contamination for each DV. There were no significant Group $\times$ Time interactions for any of the four DVs (see Table 2). There were significant main effects of time for all DVs, such that on all indices, participants reported higher scores at time 2 compared to baseline (see Table 2, Figures 1-4). One-way ANOVAs indicated there were no significant differences between groups at time 2, demonstrating that all groups were equivalent on the measures of mental contamination after the "dirty kiss" induction (see Table 2, Figures 1-4).

\subsection{Hypothesis Two - Neutralisation Behaviours}

Hypothesis 2 predicted that physical washing and mental washing would be ineffective in reducing perceived mental contamination. To test these predictions, 2 (Time; time 2, time 3) $\times 4$ (Group) ANOVAs were computed using the four indices of mental contamination as the dependent variables.

3.4.1 Feelings of dirtiness. A significant main effect of time was found for feelings of dirtiness, though there was no significant interaction between Time $\times$ Group (see Table 3). This indicated that all groups had equally reduced feelings of dirtiness from time $2(M=3.51, S E=$ $0.15)$ to time $3(M=2.00, S E=0.12$, see Table 3, Figure 1$)$.

3.4.2 Urge to wash. A significant main effect of time was found for urge to wash, though there was no significant interaction between Time $\times$ Group. This indicated that all groups had an equally reduced urge to wash from time $2(M=2.50, S E=0.15)$ to time $3(M=$ 1.42, $S E=0.08$, see Table 3, Figure 2).

3.4.3 Internal negative emotions. The results of the ANOVA revealed a significant interaction between Time $\times$ Group. The simple effect of time for each group demonstrated that there were reductions in internal negative emotions in all groups. There was a significant 
simple effect of experimental group at time 3, with post hoc tests indicating that participants in the physical washing condition completed the experiment with higher levels of internal negative emotions than both the mental washing group $\left(M_{\mathrm{diff}}=1.51, p=.001,95 \% C I[-2.53\right.$ to $-0.48])$, and the control condition $\left(M_{\text {diff }}=1.25, p=.007,95 \% C I[-2.24,-0.26]\right.$, see Table 3 , Figure 3).

3.4.4 External negative emotions. A significant Group $\times$ Time interaction effect was shown on external negative emotions. The simple effect of time for each group demonstrated that there were reductions in external negative emotions in all groups. There were significant differences between the groups at time 3. The physical washing group reported higher time 3 external negative emotion than the mental washing group $\left(M_{\mathrm{diff}}=1.42, p=.005,95 \% C I[-2.50\right.$, $-0.34])$, and the control condition $\left(M_{\text {diff }}=1.16, p=.02,95 \% C I[-2.20,-0.11]\right)$ (see Table 3, Figure 4).

\subsection{Hypothesis Three - Impact of Ineffective Neutralisation}

To assess whether final levels of internal negative emotions were related to the effectiveness of participants' neutralising actions (physical washing, mental washing, etc.), we ran a hierarchical multiple linear regression, in which baseline levels of internal negative emotion were entered as the first step, followed by change in perceived dirtiness (time 2 to time 3) at step 2. Baselines levels of internal negative emotion were not related to final intensity of internal negative emotion $\left(R^{2}=.009, F_{(1,78)}=0.72, p=.40\right)$. In contrast, the effectiveness of a participant's neutralising action predicted their final levels of internal negative emotion, with ineffectiveness of neutralisation being associated with more negative emotions at the end of the experiment $\left(\Delta R^{2}=.16, F_{(1,77)}=14.79, p<.001\right)$.

A similar multiple regression was conducted using the final scores of external negative emotions as the predicted variable. Baseline levels of external negative emotion were unrelated to final levels of external negative emotion $\left(R^{2}<.000, F_{(1,78)}<0.00, p=.99\right)$. Once 
again, however, the effectiveness of a participant's neutralising action predicted their final levels of external negative emotion, with ineffectiveness of neutralisation being associated with more intense external negative emotions at the end of the experiment $\left(\Delta R^{2}=.16, F_{(1,77)}=\right.$ 14.87, $p<.001)$.

\subsection{Discussion}

This research extends the knowledge and understanding of mental contamination, as well as the efficacy of contamination-reducing behaviours. Consistent with previous research using the “dirty kiss” paradigm (Elliott \& Radomsky, 2009; Fairbrother et al., 2005; Herba \& Rachman, 2007; Rachman et al., 2012), our use of a similar audio recording evoked mental contamination, operationalised as a perceptible increase in internal negative emotions (e.g., ashamed, guilty), external negative emotions (e.g., disgusted, angry), feelings of dirtiness, and an urge to wash. Once mental contamination was established, four experimental groups were utilised in order to examine the effects of physical washing, imagined washing, and atonement on mental contamination.

\subsection{Evoking Mental Contamination}

The finding that perpetrators of non-consensual acts experience negative emotions is consistent with Rachman’s early conceptualisation of mental contamination (1994, 2004, 2006), more recent findings examining perpetrators of the “dirty kiss” (Rachman et al., 2012) and clinical observations of perpetrators of violent crimes (Evans et al., 2007). It accords with previous research that suggests mental contamination may be elicited by a wide range of stimuli/experiences, including acting in a way that transgresses one's own ethical/moral code.

\subsection{Effect of Neutralisation Strategies}

All four groups, including the control condition, demonstrated reduction in mental contamination during the neutralisation phase of the experiment, suggesting spontaneous decay 
in feelings of contamination. None of the active neutralisation strategies were more effective than the control group in reducing perceived dirtiness or urge to wash. This is consistent with previous observations that strategies such as physical washing have been ineffective in attenuating feelings of mental contamination (Coughtrey et al., 2012; Fairbrother et al., 2005; Fairbrother \& Rachman, 2004; Fayard et al., 2009), and also with studies showing that spontaneous reductions in mental contamination occur in non-clinical samples (Coughtrey, Shafran, \& Rachman, 2014).

The poorest outcomes were associated with the physical washing task, where participants used mouthwash as their neutralisation behaviour after the “dirty kiss”. Although this group showed no differences to other conditions on measures of perceived dirtiness or urge to wash, their final levels of internal and external negative emotions were higher than the control and atonement groups. This suggests that not only is physical washing ineffective at reducing mental contamination, but that its use as a neutralisation strategy may be associated with other adverse effects such as the persistence of mental contamination that would normal spontaneously decay (Coughtrey et al., 2014).

\subsection{Impact of Ineffective Neutralisation Efforts}

Poorer outcome on measures of negative emotion was predicted to result when neutralisation strategies were ineffective. Regardless of a participant's baseline level of negative emotion, if their neutralisation behaviour was ineffective in reducing perceived dirtiness, then they were more likely to complete the task with higher levels of internal and external negative emotion. Participants who did not report a reduction in perceived dirtiness continued to experience higher levels of internal and external negative emotions. Our results accord with previous research that has observed increased levels of negative emotion during mental contamination (e.g., Radomsky \& Elliott, 2009), but is the first to demonstrate the impact of neutralisation behaviours on these emotions. 


\subsection{A Model of Neutralisation in Mental Contamination}

Our results, when integrated with previous research into mental contamination, suggest a model as depicted in Figure 5. In this model, individuals experience perceived mental contamination in response to a range of different triggering stimuli (including transgressions of one’s own values/moral code), mental events, or acting as the perpetrator of a perceived immoral act. The aversive state of mental contamination motivates the individual to seek out a neutralisation strategy aimed at reducing this. Selection of the neutralisation strategy may be complicated by difficulty in differentiating mental from contact contamination. When an individual's ability to differentiate physical from mental contamination is poor, this may lead to the selection of 'decontamination’ strategies that may be more appropriately applied to physical contaminants. The selection of strategies such as physical washing may be based on previous successful experience in using washing to deal with perceived contact contamination.

The use of strategies that have previously been effective in dealing with perceived contamination is done with the expectancy that these will serve to reduce perceived mental contamination. When these behaviours are ineffective (or their effectiveness is difficult to ascertain due to the absence of unambiguous visual indications as in mental contamination) this leads to perceived failure of the neutralisation strategy, which in turn exacerbates negative emotions. Ongoing use of ineffective strategies (e.g., washing) may persist due to their continuing effectiveness at dealing with physical contaminants, and ongoing poor differentiation of physical and mental contamination.

\subsection{Research, Clinical, and Theoretical Implications}

We believe that our results have implications for future experimental methods to investigate mental contamination, as well as for understanding the role of mental contamination in clinical conditions. Firstly, our experimental method was successful in inducing mental contamination in our participants. The use of this method, with the participant 
as perpetrator, has been less common in previous research and our results demonstrate that this method can be effectively used in non-clinical participants in future mental contamination research. Our research is the first we are aware of to make use of a selection of strategies to attempt to reduce experimentally induced mental contamination. This method may allow other research in future to examine the effects of different strategies in managing mental contamination.

Secondly, our results have implications for the understanding of mental contamination in clinical conditions outside the laboratory. It highlights the potential importance of mental contamination, and the need to ensure that exposure-based interventions elicit this perceived mental contamination as part of the exposure process. The decline in mental contamination observed in our study in the control group (and all experimental conditions) provides preliminary evidence that mental contamination may follow a similar time course to anxiety/fear during exposure-based treatment procedures, namely an initial increase on exposure, followed by habituation. It may also suggest that the use of compulsions aimed at reducing mental contamination may be counterproductive, exacerbating negative emotions. Treatments involving exposure to mental contamination should incorporate a voluntary response prevention component, in which individuals are encouraged to refrain from ineffective neutralisations. It should be noted however, than the rapid spontaneous decay of mental contamination observed in the current study, and previous research using non-clinical samples (Coughtrey et al., 2014) may contrast with clinical reports of the longevity of mental contamination (e.g., Rachman, 2006).

Finally, our results have implications for the broader understanding of the nature of the construct of mental contamination. The model of mental contamination and neutralisation strategies presented here is a first attempt to synthesise our results with those of previous authors. It attempts to integrate previous observations such as poor differentiation of mental 
and contact contamination, expectations of the effectiveness of physical washing, selection of ineffective neutralisation strategies, and the emotional impact of the ineffectiveness of these strategies (e.g., Fairbrother \& Rachman, 2004; Fairbrother, Newth, \& Rachman, 2005).

\subsection{Limitations}

There are several limitations to the current study which we hope will be addressed in future research into mental contamination. Firstly, our sample was drawn from undergraduate psychology students with low levels of obsessive-compulsive symptoms. Although the use of non-clinical samples is common in research into basic cognitive processes in OCD (Abramowitz, Fabricant, Taylor, Deacon, McKay, \& Storch, 2014), it is not known whether similar results would be observed in individuals for whom mental contamination has clinical significance. Secondly, our experimental method utilised a relatively simple set of neutralisation strategies, which may not be identical to those used by individuals with clinical levels of mental contamination problems, either in method or duration. Thirdly, our study did not use the most well validated measure of mental contamination, the Vancouver Obsessive Compulsive Inventory Mental Contamination scale (Thordarson, Radomsky, Rachman, Shafran, Sawchuk, \& Hackstian, 2004). Fourthly, although a check was conducted to examine how vividly each participant was able to visualise the dirty kiss contamination induction method, we did not assess how vivid the imagery of the mental washing task or atonement task was.

Conceptually, our research does not address a central issue of why individuals who experience mental contamination persist with ineffective neutralisation strategies, particularly if these elicit increased intensity of negative emotions as seen in the current study. It may be that individuals persist with cleaning behaviours that are effective in different contexts (e.g., dealing with contact contamination), due to poor differentiation of physical and mental contamination. Alternatively, it may be that the process of habituation that occurs over time 
(as seen in our control and intervention conditions) is mistaken for effectiveness of the cleaning behaviour, reinforcing its use.

\subsection{Future Directions}

The perpetuating mechanisms of washing behaviours seen following mental contamination remain relatively unexplored. Research in both clinical and non-clinical samples is needed in order to facilitate the development of effective experimental methods, and efficacious treatment methods. Replication of our study using clinical samples, ideographically generated mental contamination tasks, and more ecologically valid neutralisation tasks would strengthen the findings contained herein. 


\section{Declaration of Interest}

The authors have no conflict of interest. There are no fees and grants from, ownership of, employment by, and any other financial or other close relationship with an organization whose interests may be affected by the publication of the paper. 


\section{References}

Abramowitz, J.S., Fabricant, L.E., Taylor, S., Deacon, B.J., McKay, D., \& Storch, E.A. (2014). The relevance of analogue studies for understanding obsessions and compulsions. Clinical Psychology Review, 34, 206-217.

Alarcon, R.D., Libb, J.W., \& Spitler, D. (1993). A predictive study of obsessive-compulsive disorder response to clomipramine. Journal of Clinical Psychopharmacology, 13, 210-213. DOI: 10.1097/00004714-199306000-00010

Boschen, M.J. (2008). Publication trends in individual anxiety disorders: 1980-2015. Journal of Anxiety Disorders, 22, 570-575. doi:10.1016/j.janxdis.2007.04.004

Boschen, M.J., \& Drummond, L.M. (2012). Community treatment of severe, refractory obsessive-compulsive disorder. Behaviour Research and Therapy, 50, 203-209. DOI: 10.1016/j.brat.2012.01.002

Boschen, M.J., Drummond, L.M., \& Pillay, A. (2008). Treatment of severe, treatmentrefractory obsessive-compulsive disorder: A study of inpatient and community treatment. CNS Spectrums, 13, 1056-1065.

Boschen, M.J., Drummond, L.M., Pillay, A., \& Morton, K. (2010). Predicting outcome of treatment for severe, treatment resistant OCD in inpatient and community settings. Journal of Behavior Therapy an Experimental Psychiatry, 41, 90-95. doi:10.1016/j.jbtep.2009.10.006

Coehlo, J.S., \& Whittal, M.L. (2001). Are sub-types of OCD differentially responsive to treatment? Vancouver, Canada: World Congress of Behavioural and Cognitive Therapies.

Coughtrey, A.E., Shafran, R., Lee, M., \& Rachman, S.J. (2012). It's the feeling inside my head: A qualitative analysis. Behavioural and Cognitive Psychotherapy, 40, 163173. DOI:10.1017/S1352465811000658 
Coughtrey, A.E., Shafran, R., Lee, M., \& Rachman, S. (2013). The treatment of mental contamination: A case series. Cognitive and Behavioural Practice, 20, 221-231. DOI: 10.1016/j.cbpra.2012.07.002

Coughtrey, A.E., Shafran, R., \& Rachman, S.J. (2014). The spontaneous decay and persistence of mental contamination: An experimental analysis. Journal of Behavior Therapy and Experimental Psychiatry, 45, 90-96. DOI: 10.1016/j.jbtep.2013.09.001.

Drummond, L.M., Boschen, M.J., Cullimore, J., Khan-Hameed, A., White, S., \& Ion, R. (2012). Physical complications of severe, chronic obsessive-compulsive disorder: A comparison with general psychiatric inpatients. General Hospital Psychiatry, 34, 618-625. DOI: 10.1016/j.genhosppsych.2012.02.001

Elliott, C.M., \& Radomsky, A.S. (2009a). Analyses of mental contamination: Part I, experimental manipulations of morality. Behaviour Research and Therapy, 47, 9951003. DOI: 10.1016/j.brat.2009.03.004

Elliott, C.M., \& Radomsky, A.S. (2009b). Analyses of mental contamination: Part II, individual differences. Behaviour Research and Therapy, 47, 1004-1011. DOI: 10.1016/j.brat.2009.08.004

Elliott, C.M., \& Radomsky, A.S. (2010). Reversing mental contamination: The role of appraisals. Poster presented at the Association for Behavioral and Cognitive Therapies Conference, San Francisco, USA.

Elliott, C.M., \& Radomsky, A.S. (2012). Mental contamination: The effects of imagined physical dirt and immoral behaviour. Behaviour Research and Therapy, 50, 422-427. DOI:10.1016/j.brat.2012.03.007

Evans, C., Ehlers, A., Mezey, G., \& Clark, D.M. (2007). Intrusive memories in perpetrators of violent crime: Emotions and cognitions. Journal of Consulting and Clinical Psychology, 75, 134-144. DOI: 10.1037/0022-006X.75.1.134 
Fairbrother, N., Newth, S., \& Rachman, S. (2005). Mental Pollution: Feelings of dirtiness without physical contact. Behaviour Research and Therapy, 43, 121-130. DOI: 10.1016/j.brat.2003.12.005

Fairbrother, N., \& Rachman, S. (2004). Feelings of mental pollution subsequent to sexual asault. Behaviour Research and Therapy, 42, 173-189. DOI:10.1016/S00057967(03)00108-6

Farrell, L.J., \& Boschen, M.J. (2011). Treatment outcome in adult OCD: Predictors and processes of change. Asia Pacific Journal of Counselling and Psychotherapy, 2, 8297. DOI: $10.1080 / 21507686.2010 .536915$

Fayard, J.V., Bassi, A.K., Bernstein, D.M., \& Roberts, B.W. (2009). Is cleanliness next to godliness? Dispelling old wives’ tales: Failure to replicate Zhong and Liljenquist (2006). Journal of Articles in Support of the Null Hypothesis, 6, 21-30.

Fisher, P.L., \& Wells, A. (2005). How effective are cognitive and behavioural treatments for obsessive-compulsive disorder? A clinical significance analysis. Behaviour Research and Therapy, 43, 1543-1558. DOI: 10.1016/j.brat.2004.11.007

Foa, E.B., Huppert, J.D., Leiberg, S., Langer, R., \& Kichic, R. (2002). The obsessivecompulsive inventory: Development and validation of a short version. Psychological Assessment, 14, 485-496. DOI: 10.1037//1040-3590.14.4.485

Foa, E.B., Kozak, M.J., Goodman, W.K., Hollander, E., Jenike, M., \& Rasmussen, S. (1995). DSM-IV field trial: Obsessive-compulsive disorder. American Journal of Psychiatry, 152, 90-94.

Foa, E.B., Liebowitz, M.R., Kozak, M.J., Davies, S., Campeas, R., Franklin, M.E., ...Tu, X. (2005). Randomized, placebo-controlled trial of exposure and ritual prevention, clomipramine and their combination in the treatment of obsessive-compulsive 
disorder. American Journal of Psychiatry, 162, 151-161. DOI: doi:10.1176/appi.ajp.162.1.151

Fontenelle, I.S., Fontenelle, L.F., Borges, M.C., Prazeres, A.M., Range, B.P., Mendlowicz, M.V., \& Versiani, M. (2010). Quality of life and symptom dimensions of patients with obsessive-compulsive disorder. Psychiatry Research, 179, 198-203. DOI: 10.1016/j.psychres.2009.04.005

Herba, J.K., \& Rachman, S. (2007). Vulnerability to mental contamination. Behaviour Research and Therapy, 45, 2804-2812. DOI: 10.1016/j.brat.2007.07.010

Kenrick, D.T., Keefe, R.C., Gabrielidis, C., \& Cornelius, J S. (1996). Adolescents’ age preferences for dating partners: Support for an evolutionary model of life-history strategies. Child Development, 67, 1499-1511. DOI: 10.1111/j.14678624.1996.tb01810.x

Lind, C., Boschen, M.J., \& Morrissey, S.M. (2013). Technological advances in psychotherapy: Implications for the assessment and treatment of obsessive-compulsive disorder. Journal of Anxiety Disorders, 27, 47-55. DOI: 10.1016/j.janxdis.2012.09.004

Lovibond, P.F., \& Lovibond, S.H. (1995). The structure of negative emotional states: The comparison of the depression anxiety stress scales (DASS) with the beck depression and anxiety inventories. Behaviour Research and Therapy, 33, 335-343. DOI:10.1016/0005-7967(94)00075-U

Markarian, Y., Larson, M.J., Aldea, M.A., Baldwin, S.A., Good, D., Berkeljon, A., et al. (2010). Multiple pathways to functional impairment in obsessive-compulsive disorder. Clinical Psychology Review, 30, 78-88. DOI:10.1016/j.cpr.2009.09.005

Rachman, S. (2004). Fear of contamination. Behaviour Research and Therapy, 42, 1227-1255. DOI:10.1016/j.brat.2003.10.009 
Rachman, S. (2006). Fear of contamination: Assessment and treatment. Oxford, UK: Oxford University Press.

Rachman, S., \& Hodgson, R.J. (1980). Obsessions and Compulsions. Englewood Cliffs: Prentice-Hall.

Rachman, S., Radomsky, S.A., Elliott, C.M., \& Zysk, E. (2012). Mental contamination: The perpetrator effect. Journal of Behavior Therapy and Experimental Psychiatry, 43, 587-593. DOI: 10.1016/j.jbtep.2011.08.002

Shafran, R., Thordarson, D.S., \& Rachman, S.J. (1996). Thought action fusion in obsessivecompulsive disorder. Journal of Anxiety Disorders, 10, 379-391. DOI: 10.1016/0887-6185(96)00018-7

Somers, J.M., Goldner, E.M., Waraich, P., \& Hsu, L. (2006). Prevalence and incidence studies of anxiety disorders: a systematic review of the literature. The Canadian Journal of Psychiatry, 51, 100-113.

Stewart, S., Stinnett, H., \& Rosenfeld, L.B. (2000). Sex differences in desired characteristics of short-term and long-term relationship partners. Journal of Social and Personal Relationships, 17, 843-853. DOI: 10.1177/0265407500176008

Thordarson, D.S., Radomsky, A.S., Rachman, S., Shafran, R., Sawchuk, C.N., \& Hakstian, A.R. (2004). The Vancouver Obsessional Compulsive Inventory (VOCI). Behaviour Research and Therapy, 42, 1289-1314.

Warnock-Parkes, E., Salkovskis, P.M., \& Rachman, J. (2012). When the problem is beneath the surface in OCD: The cognitive treatment of a case of pure mental contamination (2012). Behavioural and Cognitive Psychotherapy, 40, 383-399.

DOI:10.1017/S1352465812000252

Zhong, C., \& Liljenquist, K. (2006). Washing away your sins: Threatened morality and physical cleansing. Science, 313, 1451-1452. DOI:10.1126/science.1130726 
MENTAL CONTAMINATION 26 


\section{Footnote}

${ }^{1}$ This method was chosen (as opposed to an alternative, potentially more powerful method suggested by a reviewer in which the participant imagined that the boy was actually 18 years old) as it did not involve retrospectively 're-writing' events which were imagined to have already occurred. This method was selected to be consistent with reappraisal that may occur in actual moral transgressions, in which changing the factual details of past events is not possible. 
Table 1.

Descriptive statistics for age and questionnaire data across all groups $(N=80)$.

\begin{tabular}{lcccccccc}
\hline & \multicolumn{2}{c}{ Physical Washing } & \multicolumn{2}{c}{ Mental Washing } & \multicolumn{2}{c}{ Atonement } & \multicolumn{2}{c}{ Control } \\
& $M$ & $S D$ & $M$ & $S D$ & $M$ & $S D$ & $M$ & $S D$ \\
\cline { 2 - 8 } Age & 20.89 & 5.59 & 26.05 & 12.87 & 23.10 & 10.81 & 24.50 & 8.94 \\
OCI-R & 8.89 & 8.08 & 12.16 & 11.95 & 9.15 & 7.39 & 10.14 & 12.61 \\
OCI-R Washing & 0.30 & 0.43 & 0.40 & 0.69 & 0.22 & 0.47 & 0.48 & 0.93 \\
DASS Depression & 3.58 & 4.19 & 5.05 & 4.39 & 7.80 & 7.16 & 3.45 & 3.56 \\
DASS Anxiety & 2.84 & 4.34 & 3.47 & 3.39 & 4.70 & 5.59 & 5.62 & 6.25 \\
DASS Stress & 7.89 & 6.61 & 10.84 & 7.67 & 9.70 & 6.56 & 9.00 & 6.44 \\
\hline
\end{tabular}


Table 2 .

Inferential Statistics for Hypothesis 1 (Evoking Mental Contamination)

\begin{tabular}{|c|c|c|c|}
\hline Dependent Variable & Time $\times$ Group Interaction & Main Effect of Time & Simple Effect of Group at Time 2 \\
\hline Feelings of Dirtiness & $F_{(3,76)}=1.82, p=.15, \eta_{p}{ }^{2}=.07$ & $F_{(1,76)}=178.50, p<.001, \eta_{p}{ }^{2}=.70$ & $F_{(3,76)}=0.37, p=.77, \eta_{p}^{2}=.01$ \\
\hline Internal Negative Emotion & $F_{(3,76)}=0.78, p=.51, \eta_{p}{ }^{2}=.03$ & $F_{(1,76)}=323.60, p<.001, \eta_{\mathrm{p}}{ }^{2}=.81$ & $F_{(3,76)}=0.93, p=.43, \eta_{p}^{2}=.04$ \\
\hline External Negative Emotion & $F_{(3,76)}=0.61, p=.61, \eta_{p}^{2}=.02$ & $F_{(1,76)}=217.92, p<.001, \eta_{p}^{2}=.81$ & $F_{(3,76)}=0.93, p=.43, \eta_{\mathrm{p}}{ }^{2}=.04$ \\
\hline Urge to Wash & $F_{(3,76)}=0.98, p=.41, \eta_{p}{ }^{2}=.04$ & $F_{(1,76)}=55.25, p<.001, \eta_{p}^{2}=.42$ & $F_{(3,76)}=0.73, p=.54, \eta_{p}^{2}=.03$ \\
\hline
\end{tabular}


Table 3.

Inferential Statistics for Hypothesis 2 (Effect of Neutralisation)

\begin{tabular}{|c|c|c|c|c|c|}
\hline & All Groups & Control & Physical Washing & Mental Washing & Atonement \\
\hline \multicolumn{6}{|l|}{ Feelings of Dirtiness } \\
\hline Time $\times$ Group Interaction & $\begin{array}{l}F_{(3,76)}=2.63, p=.06, \eta_{p}^{2} \\
=.09\end{array}$ & & & & \\
\hline Main Effect of Time & $\begin{array}{l}F_{(1,76)}=100.12, p<.001 \\
\eta_{p}^{2}=.57\end{array}$ & & & & \\
\hline \multicolumn{6}{|l|}{ Urge to Wash } \\
\hline Time $\times$ Group Interaction & $\begin{array}{l}F_{(3,76)}=1.36, p=.26, \eta_{\mathrm{p}}{ }^{2} \\
=.05\end{array}$ & & & & \\
\hline Main Effect of Time & $\begin{array}{l}F_{(1,76)}=46.94, p<.001 \\
\eta_{p}^{2}=.47\end{array}$ & & & & \\
\hline \multicolumn{6}{|l|}{ Internal Negative Emotions } \\
\hline Time $\times$ Group Interaction & $\begin{array}{l}F_{(3,76)}=7.87, p<.001 \\
\eta_{p}^{2}=.24\end{array}$ & & & & \\
\hline Simple Effect of Time & & $\begin{array}{l}t_{(21)}=7.32, p<.001, d= \\
1.54\end{array}$ & $\begin{array}{l}t_{(18)}=4.36, p<.001, d= \\
0.69\end{array}$ & $\begin{array}{l}t_{(18)}=8.71, p<.001, d= \\
2.30\end{array}$ & $\begin{array}{l}t_{(19)}=5.40, p<.001, d= \\
1.01\end{array}$ \\
\hline Simple Effect of Group (Time3) & $\begin{array}{l}F_{(3,76)}=7.03, p<.001, \\
\eta_{p}^{2}=.22\end{array}$ & $\mathrm{~B}$ & $\mathrm{~A}, \mathrm{~B}$ & A & \\
\hline \multicolumn{6}{|l|}{ External Negative Emotions } \\
\hline Time $\times$ Group Interaction & $\begin{array}{l}F_{(3,76)}=3.95, p=.01, \eta_{p}{ }^{2} \\
=.14\end{array}$ & & & & \\
\hline Simple Effect of Time & & $\begin{array}{l}t_{(21)}=5.35, p<.001, d= \\
1.50\end{array}$ & $\begin{array}{l}t_{(18)}=4.88, p<.001, d= \\
0.70\end{array}$ & $\begin{array}{l}t_{(18)}=7.36, p<.001, d= \\
2.00\end{array}$ & $\begin{array}{l}t_{(19)}=6.13, p<.001, d= \\
1.34\end{array}$ \\
\hline Simple Effect of Group (Time3) & $\begin{array}{l}F_{(3,76)}=5.40, p=.002 \\
\eta_{p}^{2}=.18\end{array}$ & $\mathrm{~B}$ & A,B & A & \\
\hline
\end{tabular}

Note. Capital letters in Simple Effects analyses of Group at Time 3 indicate groups where the DV was significantly different in pairwise post hoc $t$-tests $(p<.05)$ 


\section{Figure Captions}

Figure 1.Rating of feelings of dirtiness over time for all groups $(N=80)$.

Figure 2.Rating of urge to wash over time for all groups $(N=80)$.

Figure 3.Rating of internal negative emotions over time for all groups $(N=80)$.

Figure 4.Rating of external negative emotions over time for all groups $(N=80)$.

Figure 5. Model of mental contamination incorporating ineffective neutralisation behaviours 


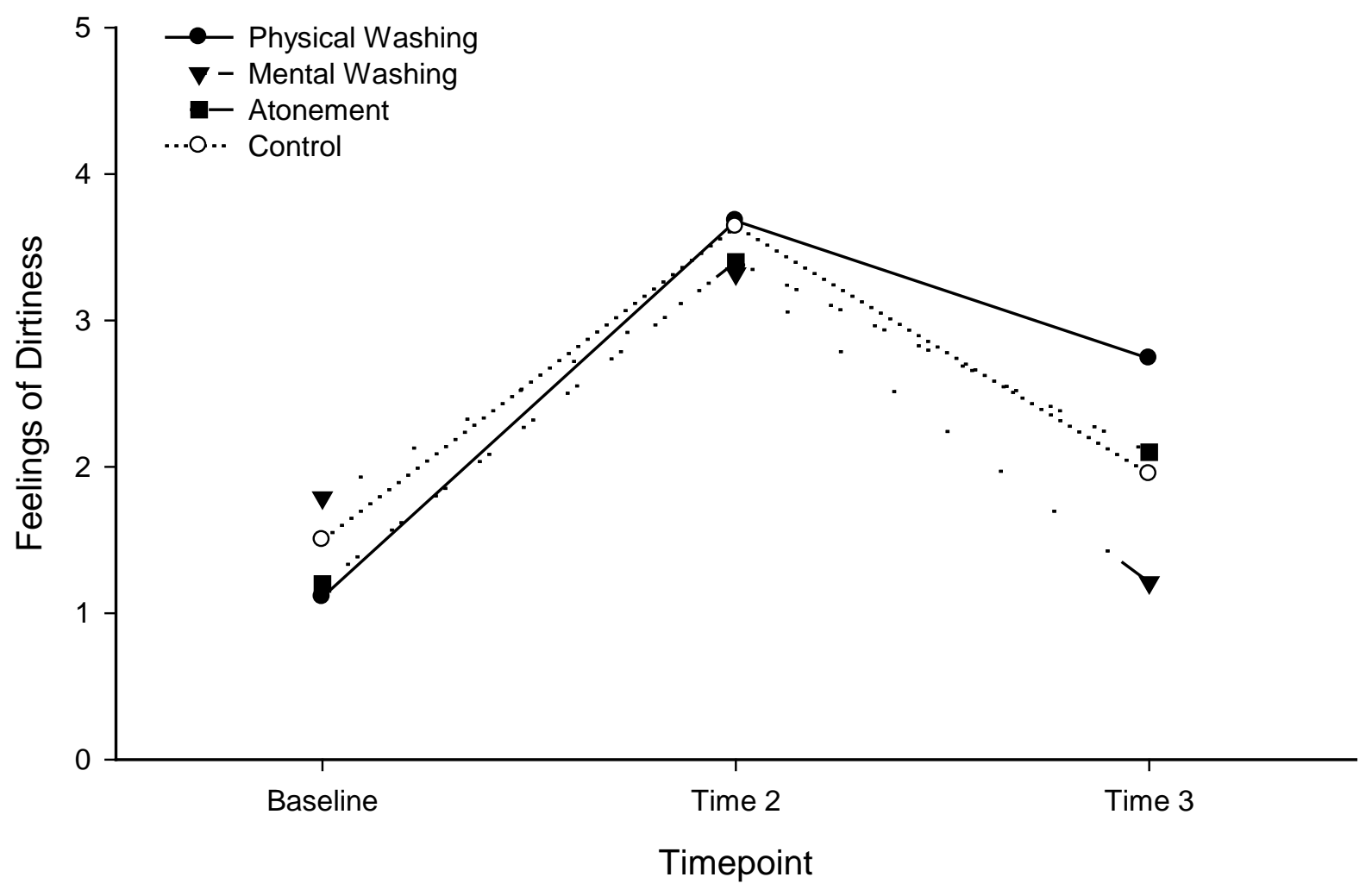




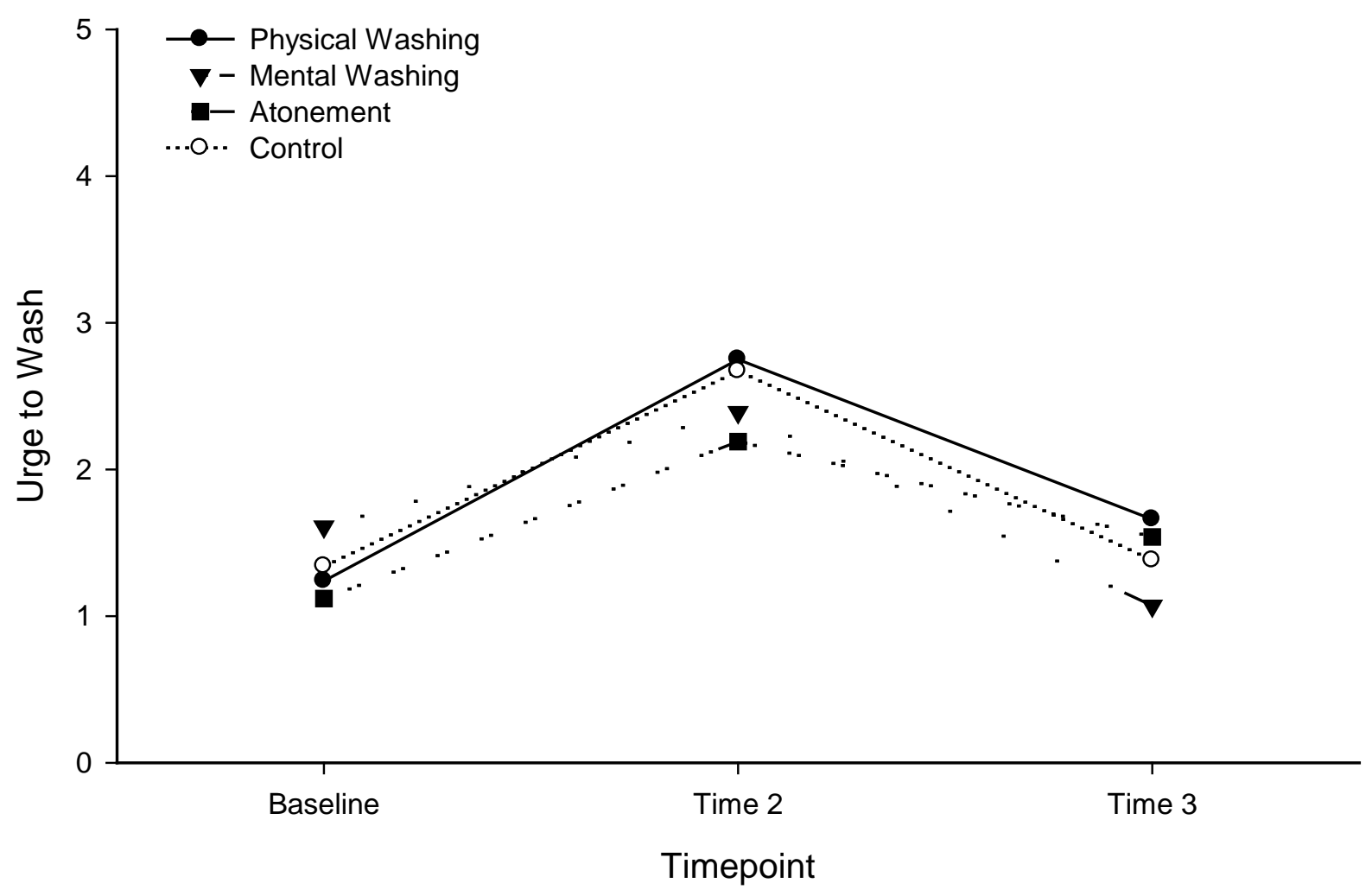




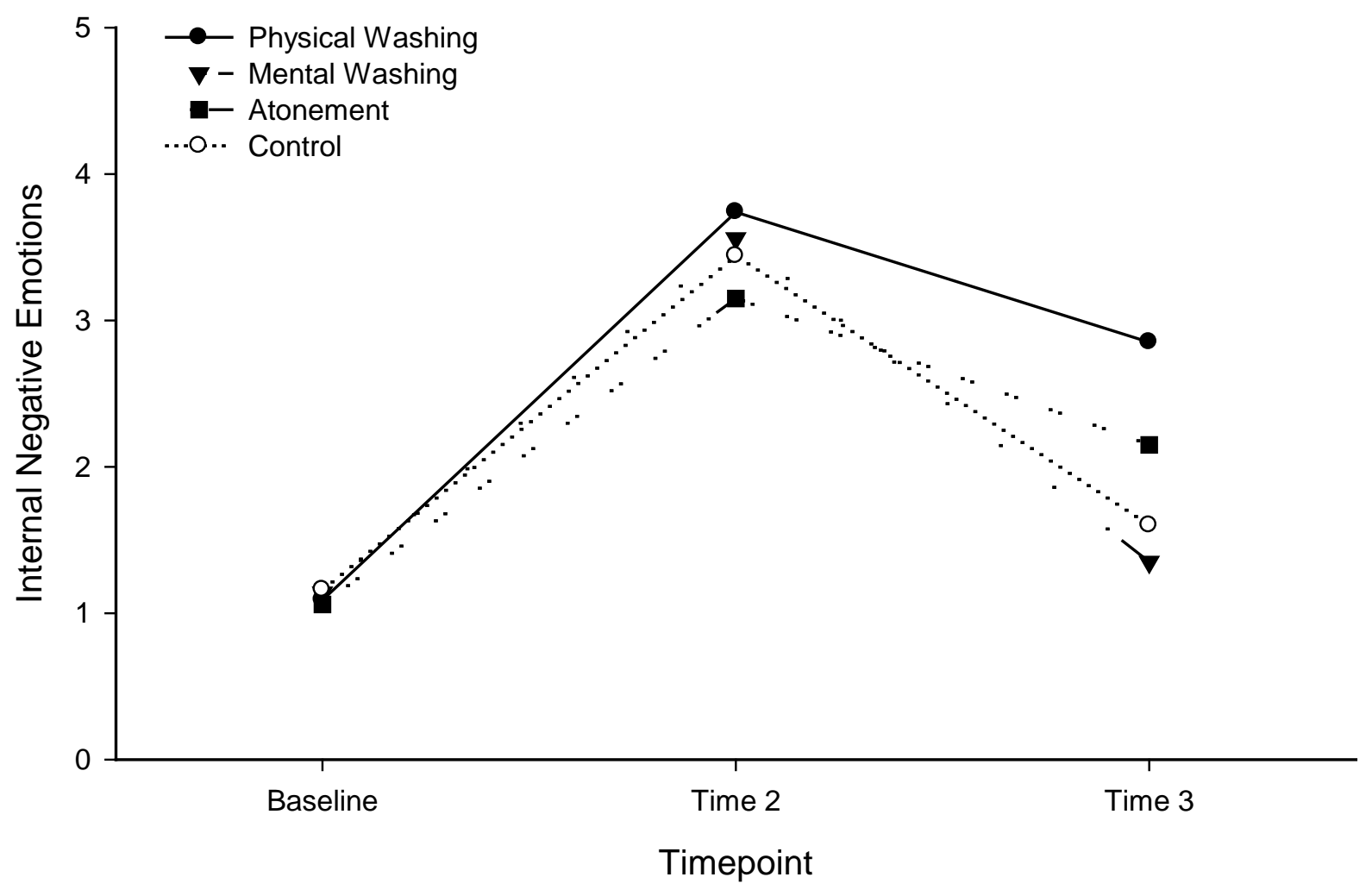




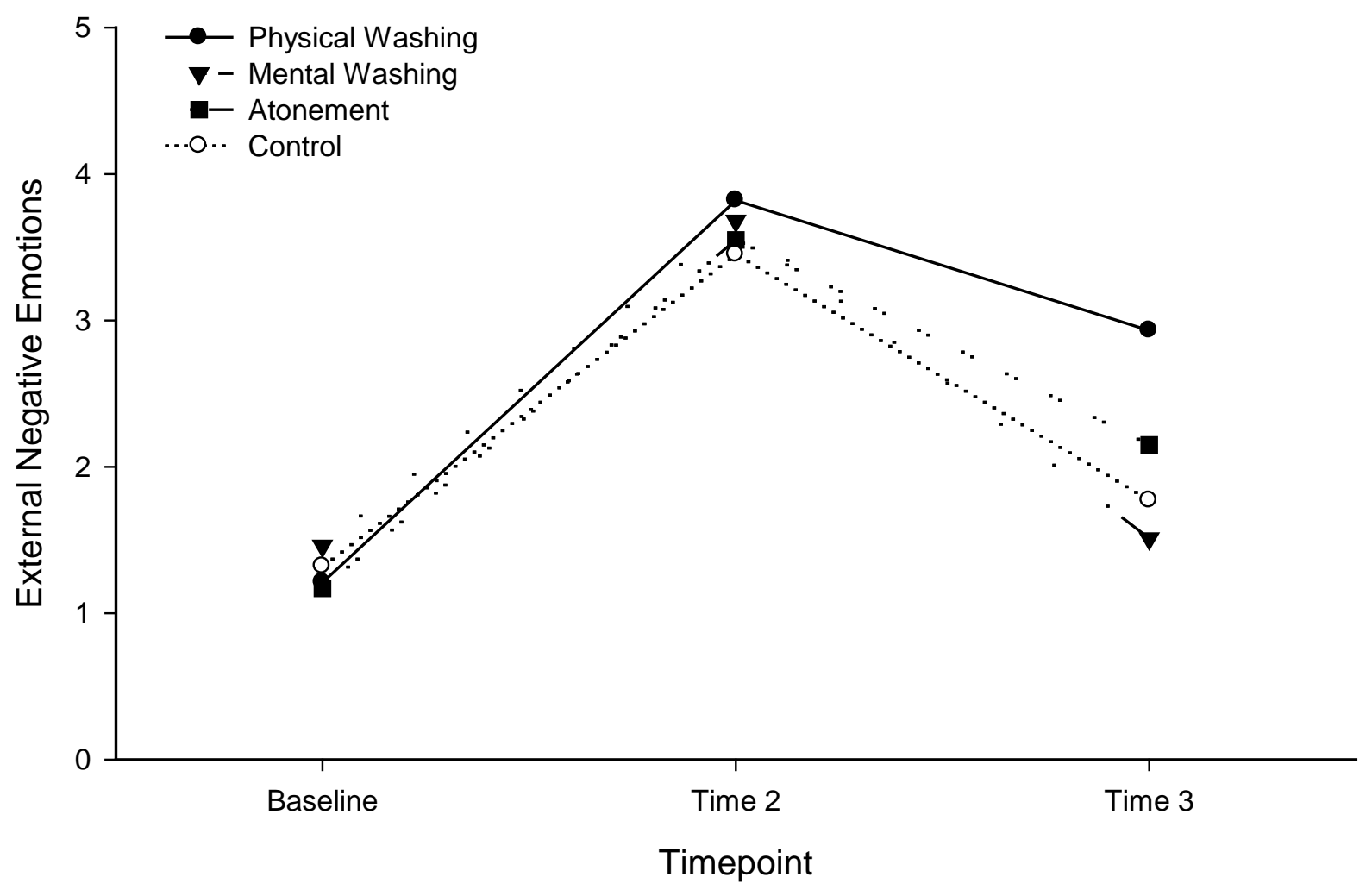




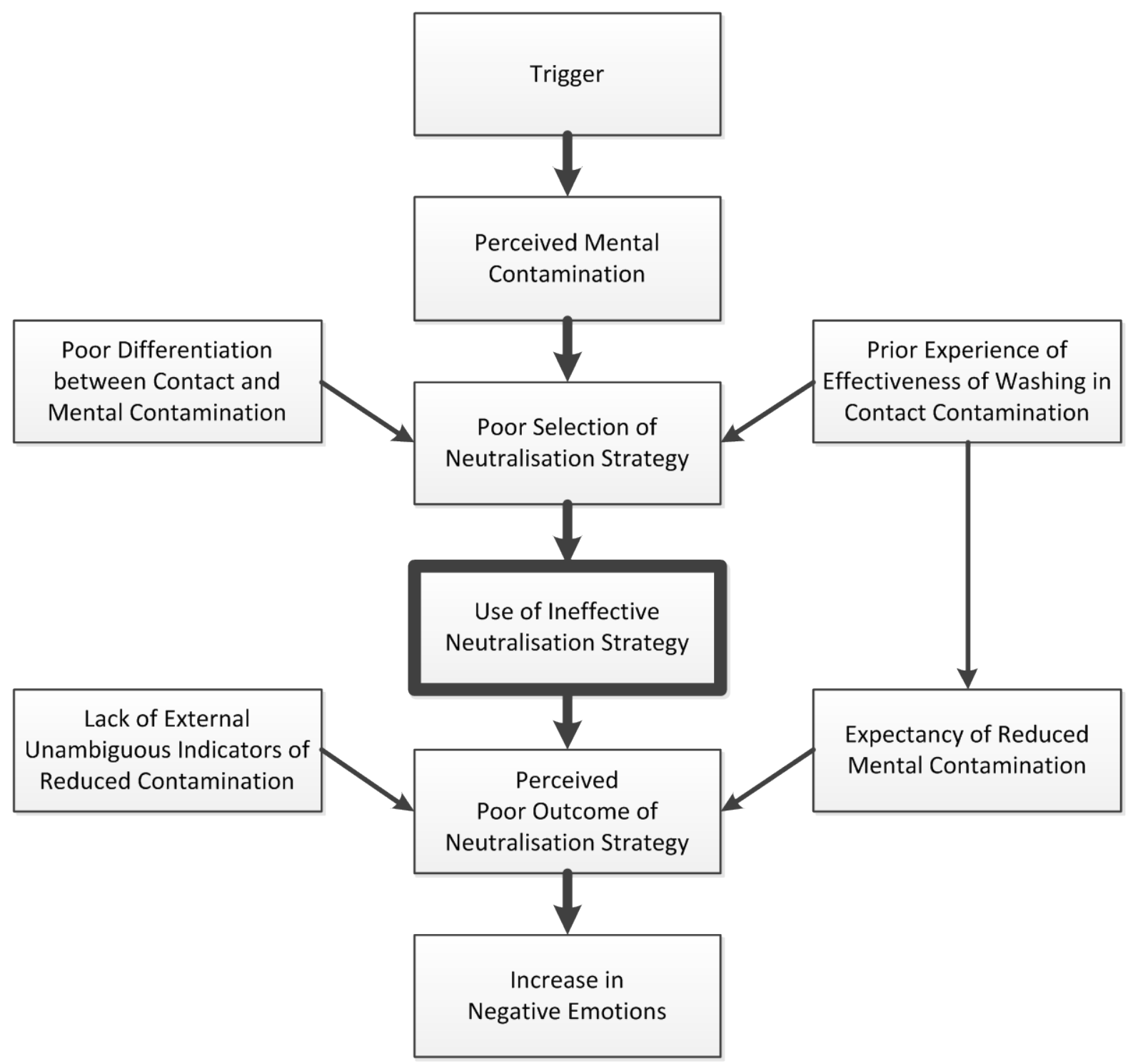

